\title{
Effects of Fungicides on Rat's Neurosteroid Synthetic Enzymes
}

\author{
Xiuwei Shen, ${ }^{1}$ Fan Chen, ${ }^{1}$ Lanlan Chen, ${ }^{2}$ Ying Su, ${ }^{2}$ Ping Huang, ${ }^{3}$ and Ren-Shan $\mathrm{Ge}^{2}$ \\ ${ }^{1}$ Department of Pharmacy, The Third Affiliated Hospital of Wenzhou Medical University, Wenzhou, Zhejiang 325200, China \\ ${ }^{2}$ Department of Anesthesiology, The Second Affiliated Hospital and Yuying Children's Hospital of Wenzhou Medical University, \\ Wenzhou, Zhejiang 325027, China \\ ${ }^{3}$ Department of Pharmacy, Zhejiang Cancer Hospital, Hangzhou, Zhejiang 310022, China
}

Correspondence should be addressed to Ren-Shan Ge; r_ge@yahoo.com

Received 16 March 2017; Revised 21 May 2017; Accepted 4 June 2017; Published 24 July 2017

Academic Editor: Jane Hanrahan

Copyright (C) 2017 Xiuwei Shen et al. This is an open access article distributed under the Creative Commons Attribution License, which permits unrestricted use, distribution, and reproduction in any medium, provided the original work is properly cited.

Exposure to environmental endocrine disruptors may interfere with nervous system's activity. Fungicides such as tebuconazole, triadimefon, and vinclozolin have antifungal activities and are used to prevent fungal infections in agricultural plants. In the present study, we studied effects of tebuconazole, triadimefon, and vinclozolin on rat's neurosteroidogenic $5 \alpha$-reductase 1 ( $5 \alpha$-Red1), $3 \alpha$ hydroxysteroid dehydrogenase ( $3 \alpha$-HSD), and retinol dehydrogenase 2 (RDH2). Rat's $5 \alpha$-Red1, $3 \alpha$-HSD, and RDH2 were cloned and expressed in COS-1 cells, and effects of these fungicides on them were measured. Tebuconazole and triadimefon competitively inhibited $5 \alpha$-Red1, with $\mathrm{IC}_{50}$ values of $8.670 \pm 0.771 \times 10^{-6} \mathrm{M}$ and $17.390 \pm 0.079 \times 10^{-6} \mathrm{M}$, respectively, while vinclozolin did not inhibit the enzyme at $100 \times 10^{-6} \mathrm{M}$. Triadimefon competitively inhibited $3 \alpha$-HSD, with $\mathrm{IC}_{50}$ value of $26.493 \pm 0.076 \times 10^{-6} \mathrm{M}$. Tebuconazole and vinclozolin weakly inhibited $3 \alpha$-HSD, with $\mathrm{IC}_{50}$ values about $100 \times 10^{-6} \mathrm{M}$, while vinclozolin did not inhibit the enzyme even at $100 \times 10^{-6} \mathrm{M}$. Tebuconazole and triadimefon weakly inhibited $\mathrm{RDH} 2$ with $\mathrm{IC}_{50}$ values over $100 \times 10^{-6} \mathrm{M}$ and vinclozolin did not inhibit this enzyme at $100 \times 10^{-6} \mathrm{M}$. Docking study showed that tebuconazole, triadimefon, and vinclozolin bound to the steroid-binding pocket of $3 \alpha$-HSD. In conclusion, triadimefon potently inhibited rat's neurosteroidogenic enzymes, $5 \alpha$-Red 1 and $3 \alpha$-HSD.

\section{Introduction}

Exposure to environmental endocrine disruptors may interfere with nervous system's activity. Fungicides such as tebuconazole (TEB) [1], triadimefon (TRI) [2], and vinclozolin (VCZ) [3] have a wide range of antifungal activities and are used to prevent fungal infections for agricultural plants. Therefore, exposure to these chemicals is very common. These fungicides contain at least one triazole or imidazole moiety in the chemical structure (Scheme 1). It is believed that these fungicides block the synthesis of fungus steroid, ergosterol. Ergosterol is a membrane component, and, therefore, these chemicals can disrupt cell membrane assembly of fungi to kill the fungi [4].

These fungicides may interfere with the steroid biosynthesis in mammals. For example, azole fungicides reduce the estrogen production via blocking aromatase $[5,6]$.
Neurosteroids are another set of steroids which have neurological activity [7]. These neurosteroids include allopregnanolone (ALLO) and $5 \alpha$-androstane- $3 \alpha, 17 \beta$-diol (DIOL) [7]. Although the classic steroids such as progesterone, estrogen, and testosterone act via binding to their respective nuclear receptors (progesterone, estrogen, and androgen receptors), neurosteroids allosterically activate the membrane GABA-A receptors and potentiate the central inhibition, causing anxiolytic, anticonvulsant, analgesic, and sedative effects $[7,8]$. GABA-A receptors are widely present in the nervous system to exert inhibitory action on nerve activity [7].

ALLO and DIOL biosynthesis requires brain $5 \alpha$-reductase 1 ( $5 \alpha$-Red1) and $3 \alpha$-hydroxysteroid dehydrogenase ( $3 \alpha$ HSD). $5 \alpha$-Redl is a smooth endoplasmic reticulum NADPHdependent enzyme [9], catalyzing progesterone or testosterone into dihydroprogesterone and dihydrotestosterone, 
<smiles>CC(C)(C)C(O)(CCc1ccc(Cl)cc1)Cn1cncn1</smiles>

Tebuconazole<smiles>CC(C)(C)C(=O)C(Oc1ccc(Cl)cc1)n1cncn1</smiles>

Triadimefon

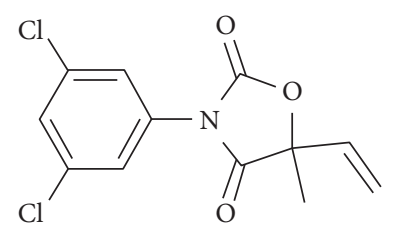

Vinclozolin

Scheme 1: The chemical structure of fungicides.

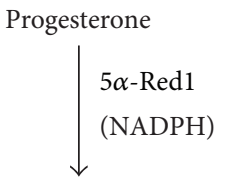

Dihydroprogesterone

$\begin{aligned} & \mathrm{RDH} 2 \\ & (\mathrm{NAD+})\end{aligned} \mid \downarrow \begin{aligned} & 3 \alpha-\mathrm{HSD} \\ & (\mathrm{NADPH})\end{aligned}$

Allopregnanolone
Testosterone

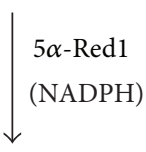

Dihydrotestosterone

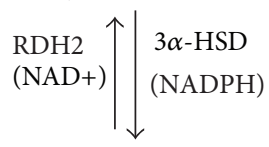

Androstanediol

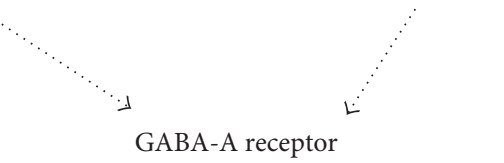

Scheme 2: The biosynthesis and metabolism of neurosteroids, allopregnanolone and androstanediol, by three distinct enzymes: NADPH-dependent $5 \alpha$-reductase 1 (SRD5A1), NADPH-dependent cytosolic $3 \alpha$-hydroxysteroid dehydrogenase (AKR1C14), and $\mathrm{NAD}^{+}$- dependent microsomal retinol dehydrogenase 2 (RDH2).

respectively [10] (Scheme 2). 3 $\alpha$-HSD, a cytosolic enzyme, catalyzes these two steroids into ALLO or DIOL, respectively [11]. In rat's brain, microsomal $\mathrm{NAD}^{+}$-dependent retinol dehydrogenase $2(\mathrm{RDH} 2)$ catalyzes the reverse reaction of ALLO or DIOL back to dihydroprogesterone and dihydrotestosterone, thus controlling the levels of these neurosteroids [12] (Scheme 2). Therefore, in the present study, we examined their direct effects on these neurosteroidogenic enzymes and their differential sensitivity.

\section{Experimental Procedures}

2.1. Chemicals. $\left[{ }^{3} \mathrm{H}\right]$ Testosterone, $\left[{ }^{3} \mathrm{H}\right]$ dihydrotestosterone, and $\left[{ }^{3} \mathrm{H}\right]$ DIOL were obtained from DuPont-New England Nuclear (Boston, MA). Testosterone, dihydrotestosterone, and DIOL were purchased from Steraloids (Newport, RI). TEB, TRI, and VCZ were purchased from Sigma-Aldrich (St. Louis, MO). TEB, TRI, and VCZ were dissolved in DMSO, which is used as a vehicle. Rat $3 \alpha$-HSD gene Akrlc14 in the expression vector $\mathrm{pRc} / \mathrm{CMV}$ was a gift from Penning T. M. (University of Pennsylvania, Philadelphia, Pennsylvania). Rat's $5 \alpha$-Red1 gene Srd5a1 and RDH2 gene Rdh2 in the expression vector pcDNA1.1 were constructed previously [13]. COS-1 cell line was purchased from ATCC (Manassas, VA).

2.2. Transient Transfection. COS-1 cells were maintained in DMEM medium (Life Technologies, Inc., Gaithersburg, MD) supplemented with $10 \%$ fetal calf serum and $5 \% \mathrm{CO}_{2}$ at $37^{\circ} \mathrm{C}$. For transfection, $1 \times 10^{6}$ cells were seeded per well in a six-well plate and cultured for $24 \mathrm{~h}$ in media supplemented with charcoal-stripped fetal calf serum to obtain $50-80 \%$ confluence. Transfection was performed by the FuGENE 6 Transfection Reagent (Roche Molecular Biochemicals, Indianapolis, IN) according to the manufacturer's protocol. $1 \mu \mathrm{g}$ DNA per well showed maximal efficiency and, therefore, this quantity was used in the transfection assays.

2.3. Preparation of $5 \alpha-R e d 1,3 \alpha-H S D$, and RDH2 Proteins. Twenty-four hours after transfection, the COS-1 cells were scraped from dishes and were homogenized in $10 \mathrm{ml} 0.01 \mathrm{mM}$ phosphate-buffered saline containing $(0.25 \mathrm{M})$ sucrose and nuclei and large cell debris were removed by centrifugation at $1500 \times \mathrm{g}$ for $10 \mathrm{~min}$. Microsomal and cytosolic fractions were harvested after subsequent centrifugation at $10,000 \times \mathrm{g}$ for $1 \mathrm{~h}$ and at $105,000 \times \mathrm{g}$ for $1 \mathrm{~h}$ twice. The protein concentrations in cell lysates and subcellular fractions were measured using a kit (number 500-0006, Bio-Rad Laboratories, Inc., Hercules, CA) with bovine serum albumin as a standard. The concentrations of rat's $5 \alpha$-Red1, $3 \alpha$-HSD, and RDH2 proteins were $20 \mathrm{mg} / \mathrm{ml}$. The proteins were used for the measurement of $5 \alpha$ Red1, $3 \alpha$-HSD, and RDH2 activities.

2.4. Measurement of $5 \alpha$-Red1, $3 \alpha-H S D$, and RDH2 Activities. $5 \alpha$-Red 1 activity was measured by incubating $1000 \mathrm{nM}$ testosterone spiked with $60,000 \mathrm{dpm}$ of $\left[{ }^{3} \mathrm{H}\right]$ testosterone as the substrate, $10 \mu \mathrm{g}$ SRD5A1-containing microsomal protein, and $0.2 \mathrm{mM}$ NADPH in $250 \mu \mathrm{l}$ PBS ( $\mathrm{pH}=7.2$ ). $3 \alpha$-HSD activity was measured by incubating $1000 \mathrm{nM}$ dihydrotestosterone spiked with $60,000 \mathrm{dpm}$ of $\left[{ }^{3} \mathrm{H}\right]$-dihydrotestosterone as the substrate, $10 \mu \mathrm{g} 3 \alpha$-HSD-containing cytosolic protein, and $0.2 \mathrm{mM}$ NADPH in $250 \mu \mathrm{l}$ PBS ( $\mathrm{pH}=7.2$ ). RDH2 activity was measured by incubating $1000 \mathrm{nM}$ DIOL spiked with $630,000 \mathrm{dpm}$ of $\left[{ }^{3} \mathrm{H}\right]$ DIOL as the substrate, $10 \mu \mathrm{g} \mathrm{RDH} 2-$ containing microsomal protein, and $0.2 \mathrm{mM} \mathrm{NAD}^{+}$in $250 \mu \mathrm{l}$ PBS ( $\mathrm{pH}=7.2) .100 \mu \mathrm{M}$ fungicides were incubated in the 
TABLE 1: The enzyme kinetic parameters and the half maximal inhibitory concentration $\left(\mathrm{IC}_{50}\right)$ of fungicides.

\begin{tabular}{lccr}
\hline Parameters & $5 \alpha$-Red 1 & $3 \alpha$-HSD & RDH2 \\
\hline Apparent $K_{m}(\mu \mathrm{M})$ & $1.397 \pm 0.35$ & $3.148 \pm 0.197$ & $2.850 \pm 0.037$ \\
Apparent $V_{\max }(\mathrm{pmol} / \mathrm{mg} \cdot \mathrm{min})$ & $3.494 \pm 0.287$ & $66.69 \pm 1.589$ & $529.5 \pm 2.612$ \\
$\mathrm{IC}(\mu \mathrm{M})$ & & & \\
Tebuconazole & $8.670 \pm 0.771$ & $\sim 100$ & $>100$ \\
Triadimefon & $17.39 \pm 0.079$ & $26.493 \pm 0.076$ & $>100$ \\
Vinclozolin & $\mathrm{NI}$ & $\sim 100$ & $\mathrm{NI}$ \\
\hline
\end{tabular}

Mean \pm SE, $n=4$. NI: no inhibition at $100 \mu \mathrm{M}$.

respective reaction mixture at $37^{\circ} \mathrm{C}$ for $60 \mathrm{~min}$ for the initial inhibition test. The inhibitory potency of fungicides was measured relative to the control (only DMSO). Each fungicide was dissolved in DMSO and an aliquot $(1 \mu \mathrm{l})$ of each fungicide was added to the reaction mixture at a final concentration of $0.4 \%$, at which concentration DMSO did not inhibit $5 \alpha$-Red1, $3 \alpha$-HSD, or RDH2 activities. The reaction was stopped with $1 \mathrm{ml}$ ice-cold ether. The steroids were extracted with ether after vigorous vortexing. The organic ether layer was transferred to the new glass tube and dried under nitrogen. The steroids were separated chromatographically on the thin layer plate in chloroform and methanol $(90: 3$, $\mathrm{v} / \mathrm{v}$ ), and the radioactivity was measured using a scanning radiometer (System AR2000, Bioscan Inc., Washington, DC) as previously described [14]. The percentage conversion of testosterone into dihydrotestosterone (for $5 \alpha$-Red1), dihydrotestosterone into DIOL (for $3 \alpha$-HSD), and DIOL into dihydrotestosterone (for $\mathrm{RDH} 2$ ) was calculated by dividing the radioactive counts identified as the respective steroids by the total counts of control DMSO.

2.5. Determination of Enzyme Kinetics. The enzyme kinetics was determined by adding $0.0315-10 \mu \mathrm{M}$ testosterone or dihydrotestosterone or DIOL for $5 \alpha$-Red1, $3 \alpha$-HSD, and RDH2. The Michaelis-Menten equation was used by GraphPad (Version 6, GraphPad Software Inc., San Diego, CA) to calculate the apparent Michaelis-Menten constant $\left(K_{m}\right)$ and the apparent maximum velocity $\left(V_{\max }\right)$. The initial velocity $\left(V_{o}\right)$ depends on the apparent $K_{m}, V_{\max }$, and the substrate concentration $([S])$ as $V_{o}=V_{\max }[S] /\left(K_{m}+[S]\right)$.

2.6. Determination of $I C_{50}$ Values and Inhibitory Modes. The half maximum inhibitory concentration $\left(\mathrm{IC}_{50}\right)$ of TEB or TRI to inhibit $5 \alpha$-Red 1 was determined by adding $1000 \mathrm{nM}$ of testosterone with $0.2 \mathrm{mM}$ NADPH and $10^{-8}-10^{-4} \mathrm{M}$ TEB or TRI in $250 \mu \mathrm{l}$ phosphate-buffered saline $(0.1 \mathrm{mM})$ containing $5 \alpha$-Red 1 protein and incubating each reaction mixture for $60 \mathrm{~min}$. The $\mathrm{IC}_{50}$ value of TRI to inhibit $3 \alpha-\mathrm{HSD}$ was determined by adding $1000 \mathrm{nM}$ of dihydrotestosterone with $0.2 \mathrm{mM}$ NADPH and $10^{-8}-10^{-4} \mathrm{M}$ TRI in $250 \mu \mathrm{l}$ phosphatebuffered saline $(0.1 \mathrm{mM})$ containing $3 \alpha$-HSD protein and incubating each reaction mixture for $60 \mathrm{~min}$. For determining the mode of inhibition of $5 \alpha$-Red $1,10^{-9}-10^{-5} \mathrm{M}$ testosterone was added to the reaction mixture in the presence of TEB $(10$ and $20 \mu \mathrm{M})$ or TRI $(20$ and $40 \mu \mathrm{M})$ for $5 \alpha$-Red1. For determining the mode of inhibition of $3 \alpha$-HSD, $10^{-9}-10^{-5} \mathrm{M}$ dihydrotestosterone was added to the reaction mixture in the presence of TRI ( 25 and $50 \mu \mathrm{M})$ for $3 \alpha$-HSD.

2.7. Preparation of Protein and Ligand Structures and Docking. The crystal structure of rat's $3 \alpha$-HSD containing $\mathrm{NADP}^{+}$ and testosterone (PDB id lafs [15]) was used as a docking target for steroid substrate DIOL, TEB, TRI, and VCZ. These chemical structures were obtained from PubChem (https://pubchem.ncbi.nlm.nih.gov) as ligands. Docking calculations were performed with SwissDock, a docking algorithm based on the docking software EADock DSS [16]. The docked file was visualized using the program Chimera 1.1.1 (San Francisco, CA) and the free energy was calculated.

2.8. Statistics. Each experiment was repeated four times. Data were subjected to a nonlinear regression analysis by GraphPad (Version 6, GraphPad Software Inc., San Diego, CA) for $\mathrm{IC}_{50}$ values. Lineweaver-Burk plot was used for the mode of inhibition. Data were subjected to an analysis by ANOVA followed by ad hoc Tukey's comparison to identify significant differences between the control (CON) and TEB, TRI, or VCZ group. All data are expressed as means \pm SEM. The difference was regarded as significant at $P<0.05$.

\section{Results}

3.1. Effects of Fungicides on $5 \alpha$-Red1. The conversion of testosterone into DHT is catalyzed by $5 \alpha$-Red1, which requires $\mathrm{NADPH}$ as a cofactor; the apparent $K_{m}$ and apparent $V_{\max }$ of $5 \alpha$-Red1 were $1.397 \pm 0.35 \mu \mathrm{M}($ mean \pm SE, $n=4)$ and $3.494 \pm$ $0.287 \mathrm{pmol}$ dihydrotestosterone $/ \mathrm{mg}$ protein $/ \mathrm{min}$ (mean $\pm \mathrm{SE}$, $n=4$ ), respectively (Table 1 and Figure $1(\mathrm{a})$ ). As presented in Figure 1(b), when the highest concentration $(100 \mu \mathrm{M})$ was tested, TEB and TRI inhibited rat's $5 \alpha$-Red1 to 26.94 $\pm 5.30 \%$ and $19.31 \pm 3.6 \%$ of the control value, respectively, but VCZ only to $74.17 \pm 5.57 \%$ of the control value. We further calculated the $\mathrm{IC}_{50}$ values of TEB (Figure 1(c)) and TRI (Figure 1(d)), which were $8.670 \pm 0.771$ and $17.390 \pm$ $0.079 \mu \mathrm{M}$, respectively (Table 1 ). The modes of inhibition of TEB and TRI on $5 \alpha$-Redl were found to be competitive against testosterone (Figures 2(a) and 2(b)).

3.2. Effects of Fungicides on $3 \alpha$-HSD Activity. The conversion of dihydrotestosterone into DIOL is catalyzed by $3 \alpha-\mathrm{HSD}$, which requires NADPH as a cofactor; the apparent $K_{m}$ and apparent $V_{\max }$ of $3 \alpha$-HSD were $3.148 \pm 0.197 \mu \mathrm{M}$ (mean \pm 


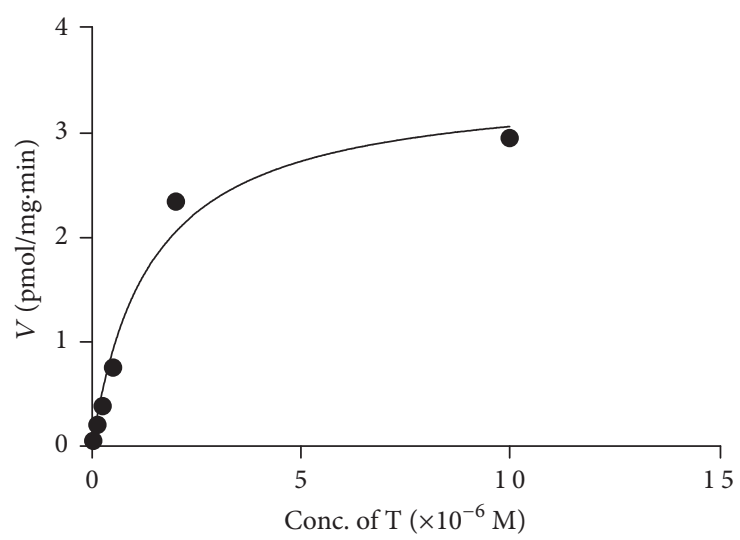

(a) $5 \alpha$-Red1

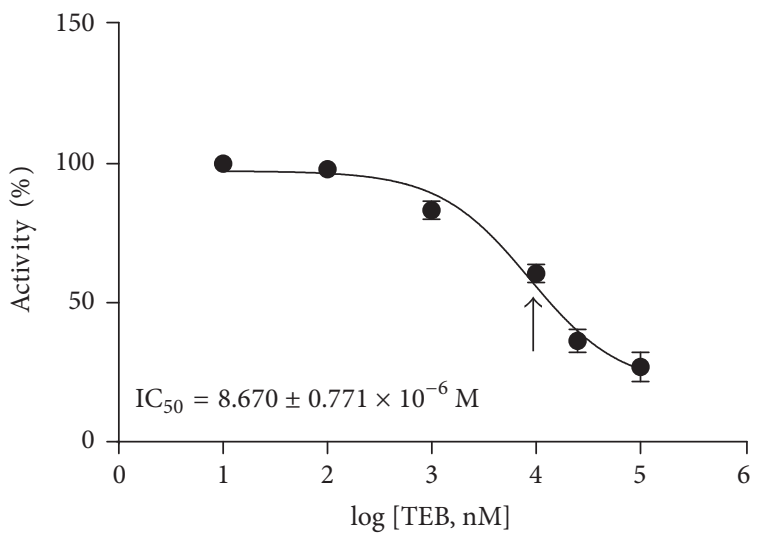

(c) $5 \alpha$-Red1:TEB

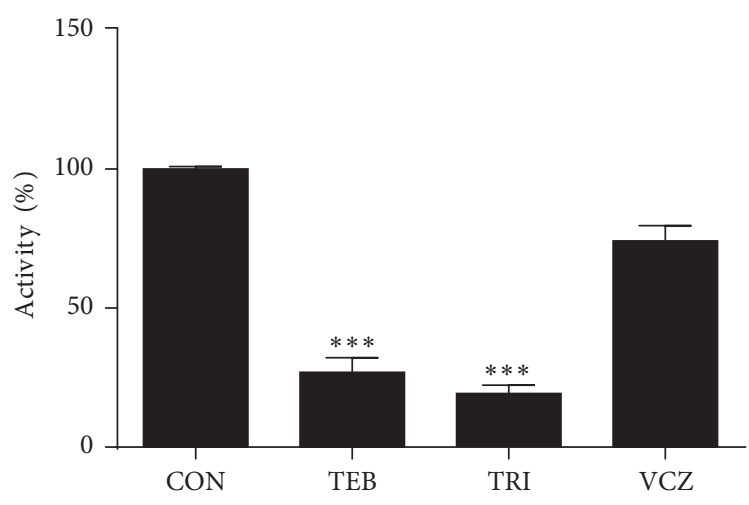

(b) $5 \alpha$-Red1

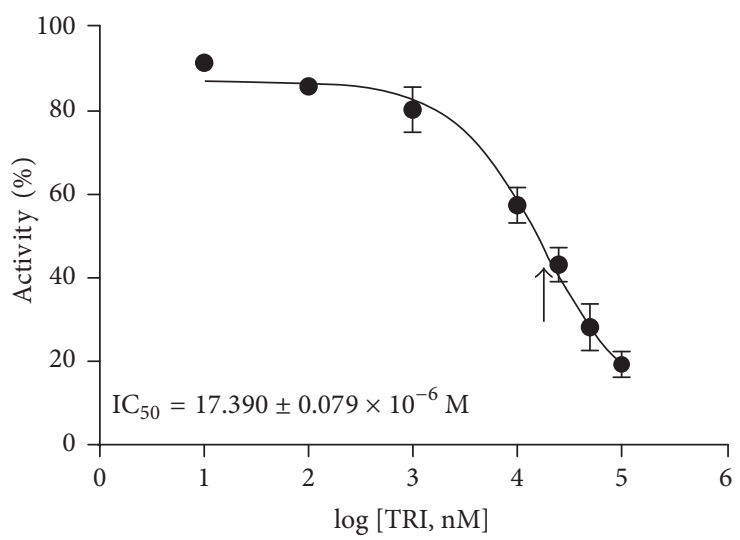

(d) $5 \alpha$-Red1:TRI

FIGURE 1: Kinetics of SRD5A1 and the inhibition of fungicides. Panel (a): kinetics of SRD5A1 with testosterone (T) as the substrate. Panel (b): $\%$ inhibition by tebuconazole (TEB), triadimefon (TRI), and vinclozolin (VCZ) at $100 \mu \mathrm{M}$. Panels (c) and (d): IC F $_{50}$ values of TEB and TRI. Mean $\pm \mathrm{SEM} ; * * *$ indicates a significant difference compared to the control $(\mathrm{CON})$ at $P<0.001$.

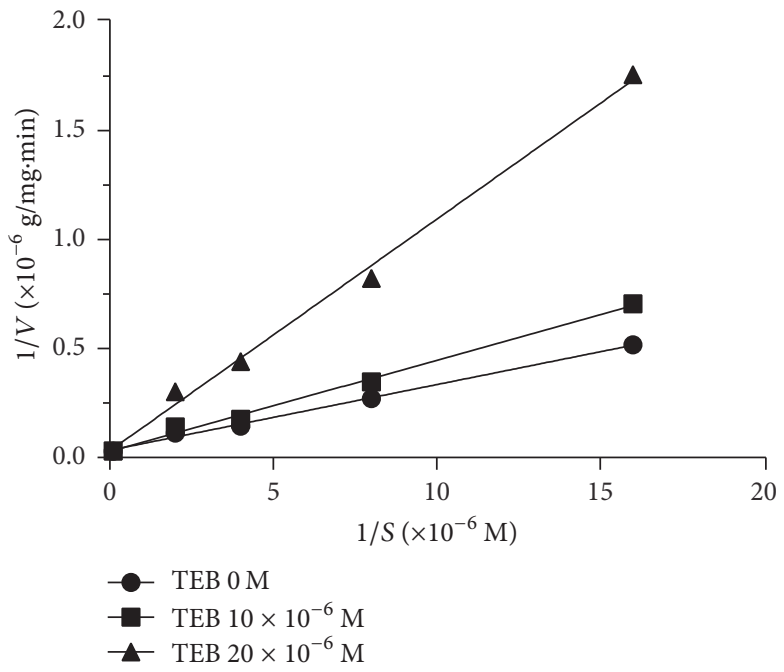

(a) TEB versus $\mathrm{T}$

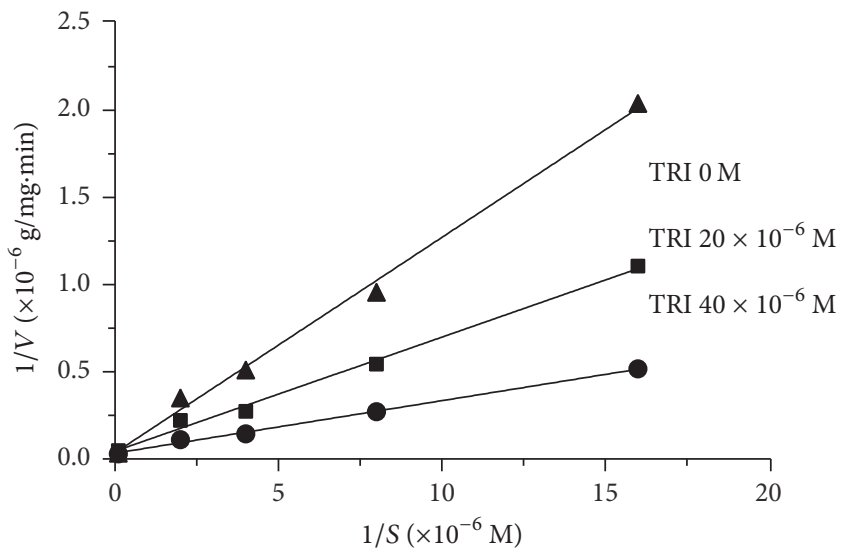

(b) TRI versus $\mathrm{T}$

FIGURE 2: The inhibitory mode of tebuconazole (TEB) and triadimefon (TRI) on rat's SRD5A1. Lineweaver-Burk plots in presence of testosterone and TEB (Panel (a)) as well as testosterone and TRI (Panel (b)). Values were obtained from four samples. 


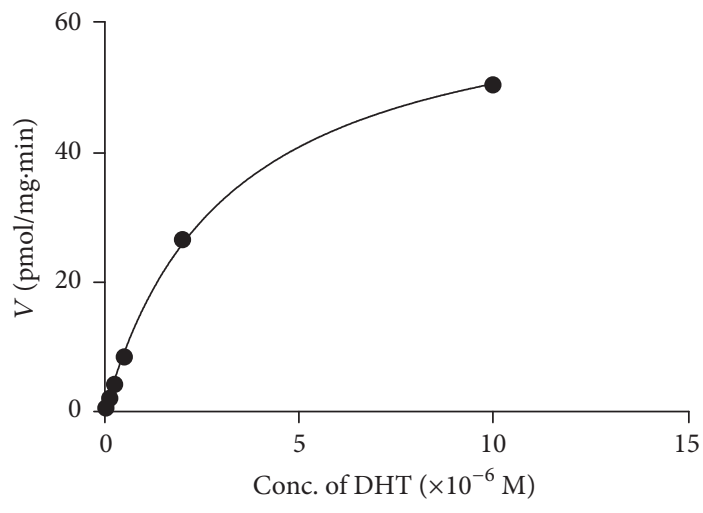

(a) $3 \alpha-\mathrm{HSD}$

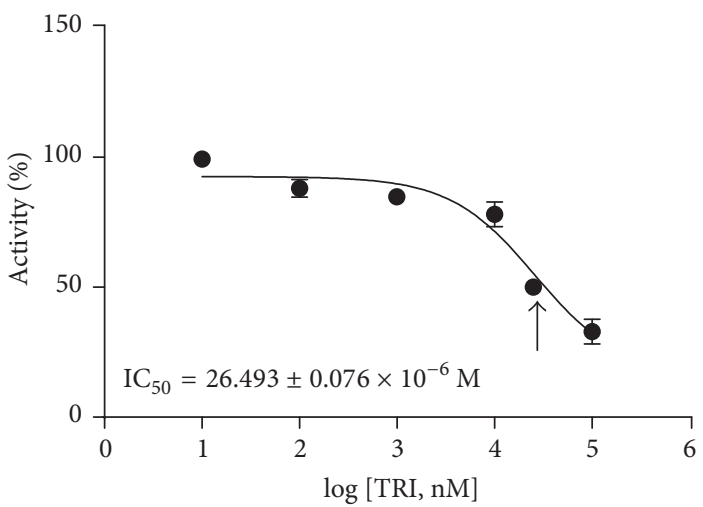

(c) $3 \alpha$-HSD:TRI

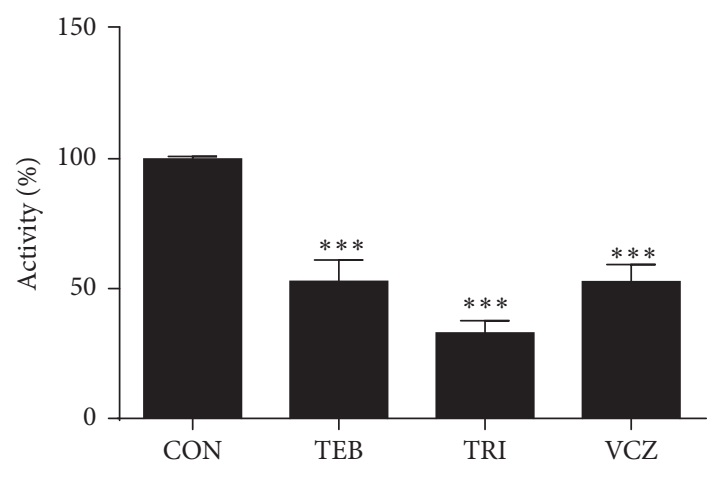

(b) $3 \alpha$-HSD

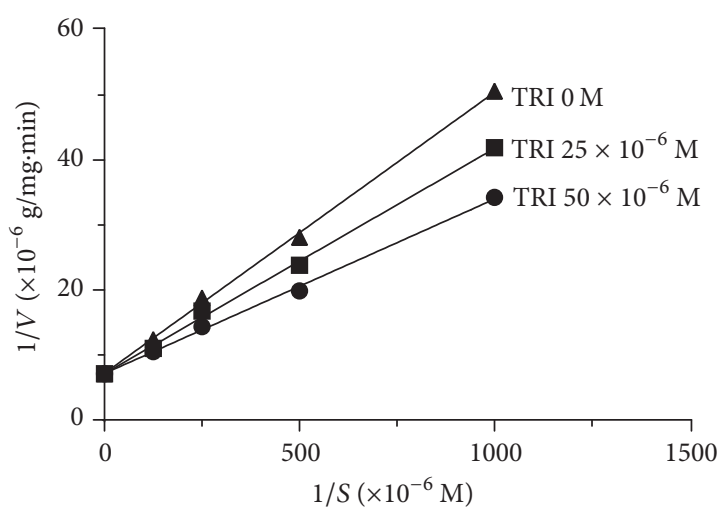

(d) TRI versus DHT

FIGURE 3: The kinetics of AKR1C14 and the inhibition of fungicides. Panel (a): kinetics of AKR1C14 with dihydrotestosterone (DHT) as the substrate. Panel (b): \% inhibition by tebuconazole (TEB), triadimefon (TRI), and vinclozolin (VCZ) at $100 \mu$ M. Panel (c): IC F $_{50}$ value of TRI. Panel (d): the mode of inhibition of TRI versus DHT. Mean \pm SEM; $* * *$ indicates a significant difference compared to the control (CON) at $P<0.001$.

SE, $n=4)$ and $66.69 \pm 1.587 \mathrm{pmol} \mathrm{DIOL} / \mathrm{mg}$ protein $/ \mathrm{min}$ (mean \pm SE, $n=4$ ), respectively (Table 1 and Figure $3(\mathrm{a})$ ). TRI inhibited rat's $3 \alpha$-HSD to $32.95 \pm 4.80 \%$ of the control value, while TEB and VCZ caused about $52.78 \pm 8.278 \%$ and 52.65 $\pm 6.70 \%$ of the control value, respectively (Figure 3(b)). We further calculated the $\mathrm{IC}_{50}$ value of TRI, which was $26.493 \pm$ $0.076 \mu \mathrm{M}$ (Table 1 and Figure 3(c)). The mode of inhibition of TRI on $3 \alpha$-HSD was found to be competitive against dihydrotestosterone (Figure 3(d)).

3.3. Effects of Fungicides on RDH2 Activity. The conversion of DIOL into dihydrotestosterone is catalyzed by $\mathrm{RDH} 2$, which requires $\mathrm{NAD}^{+}$as a cofactor; the apparent $K_{m}$ and apparent $V_{\max }$ of RDH2 were $2.850 \pm 0.037 \mu \mathrm{M}$ (mean $\left.\pm \mathrm{SE}, n=4\right)$ and $529.5 \pm 2.612 \mathrm{pmol}$ dihydrotestosterone $/ \mathrm{mg}$ protein $/ \mathrm{min}$ (mean \pm SE, $n=4$ ), respectively (Table 1 and Figure 4(a)). TRI and TEB only inhibited RDH2 to $65.79 \pm 1.69 \%$ and $53.35 \pm 5.03 \%$ of the control value, while VCZ did not inhibit the enzyme activity $(85.51 \pm 2.20 \%$ of the control value, Figure 4(b)).

3.4. Docking of Fungicides to $3 \alpha$-HSD. Because among three enzymes only the crystal structure of rat's $3 \alpha$-HSD is available, we docked DIOL to $3 \alpha$-HSD first. DIOL was found to bind to the dihydrotestosterone-binding pocket, with free energy of $-7.73 \mathrm{Kcal}$. Further docking analysis for TEB (Figure 5(a)), TRI (Figure 5(b)), and VCZ (Figure 5(c)) showed that all these three chemicals bound to the steroid-binding pocket, with free energies of $7.28,-7.63$, and -7.34 . These data indicate that TRI has the highest binding affinity with $3 \alpha$-HSD. TRI interacts with Try310, Trp227, His117, Tyr55, Leu54, Thr24, and Asn306 residues of $3 \alpha$-HSD (Figure 6). The Tyr310 and Trp227 residues were believed to hold the steroid structure, and His117 and Tyr55 residues were believed to catalyze the $3 \alpha$-position of the steroid [15].

\section{Discussion}

In the brain, the neurosteroidogenic enzymes $5 \alpha$-Red1 [17], $3 \alpha$-HSD [11, 17], and RDH2 [12] are involved in the biosynthesis and metabolism of neurosteroids. $5 \alpha$-Red 1 and $3 \alpha$-HSD are responsible for the neurosteroid biosynthesis to form $3 \alpha$ reduced neurosteroids, while $\mathrm{RDH} 2$ is responsible for the neurosteroid metabolism to remove the $3 \alpha$-reduced neurosteroids. These neurosteroidogenic enzymes showed different sensitivity to some fungicides. Here, we demonstrated that TEB and TRI potently inhibited $5 \alpha$-Red1, the irreversible step of neurosteroid biosynthesis. Furthermore, TRI also 


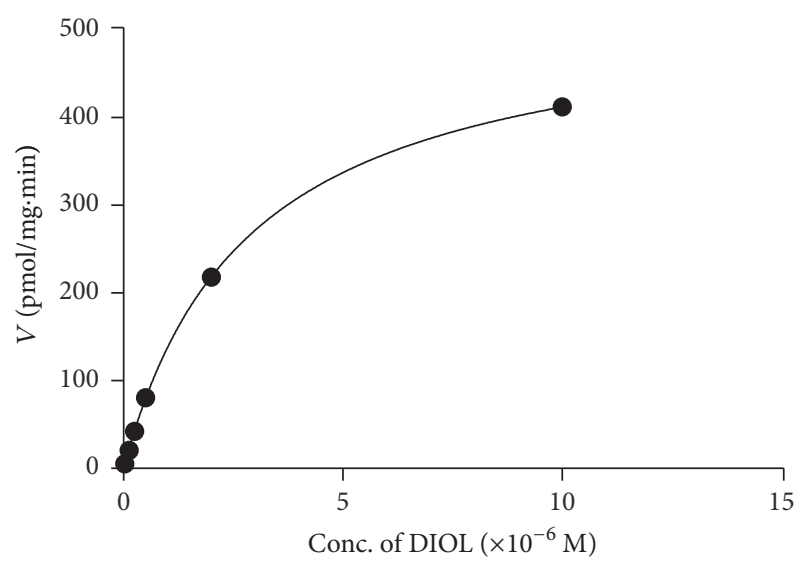

(a) $\mathrm{RDH} 2$

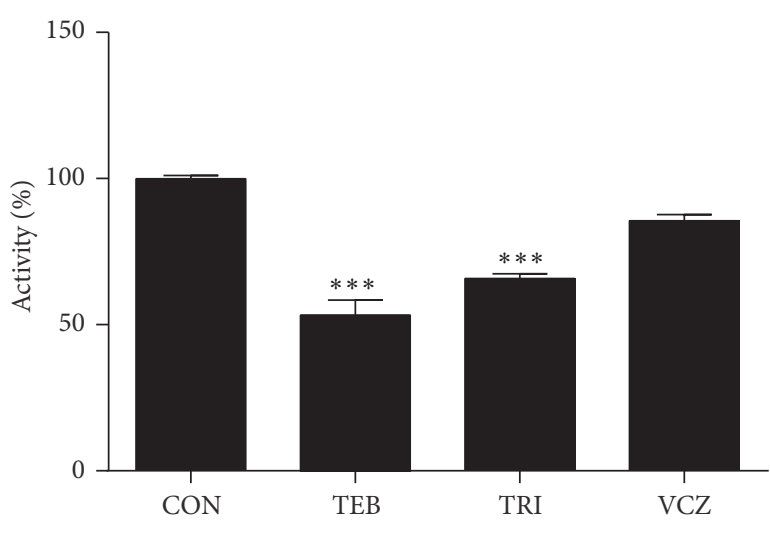

(b) $\mathrm{RDH} 2$

Figure 4: The kinetics of RDH2 and the inhibition of fungicides. Panel (a): kinetics of RDH2 with androstanediol (DIOL) as the substrate. Panel (b): \% inhibition by tebuconazole (TEB), triadimefon (TRI), and vinclozolin (VCZ) at $100 \mu \mathrm{M}$. Mean \pm SEM; *** indicates a significant difference compared to the control at $P<0.001$.

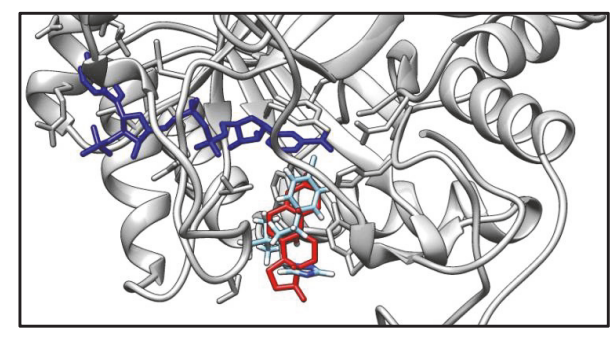

(a)

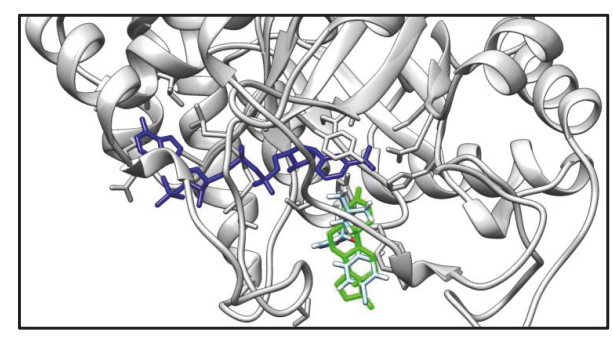

(b)

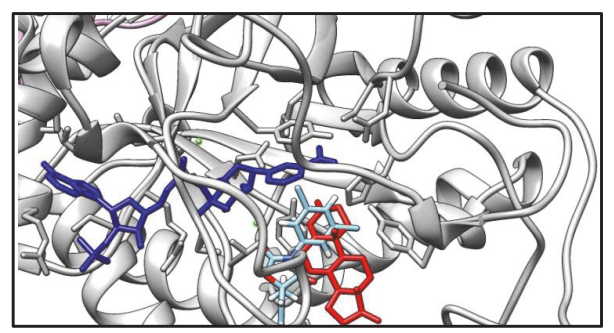

(c)

FIGURE 5: Docking analysis for the binding to rat's AKR1C14 (1AFS). Panel (a): tebuconazole; blue structure, NADPH; red structure, testosterone; sky-blue structure, tebuconazole. Panel (b): triadimefon; blue structure, NADPH; green structure, dihydrotestosterone; skyblue structure, triadimefon. Panel (c): vinclozolin; blue structure, NADPH; red structure, testosterone; sky-blue structure, vinclozolin.

potently inhibited $3 \alpha$-HSD, thus leading to the reduced level of neurosteroids. VCZ was the weakest fungicide to inhibit $5 \alpha$-Red 1 and $3 \alpha$-HSD.

Interestingly, the enzyme $5 \alpha$-Red 1 is the most sensitive to the inhibition by TEB compared to $3 \alpha$-HSD and RDH2. The $\mathrm{IC}_{50}$ values of TEB for $5 \alpha$-Red1, $3 \alpha$-HSD, and RDH2 were $8.67, \sim 100$, and $\sim 100 \mu \mathrm{M}$. $5 \alpha$-Red 1 and $3 \alpha$-HSD share equal sensitivity to the inhibition by TRI compared to RDH2. The $\mathrm{IC}_{50}$ values of TRI for $5 \alpha$-Red1, $3 \alpha$-HSD, and RDH 2 were $17.39,26.49$, and $\sim 100 \mu \mathrm{M}$. The reason for this difference is still unclear. This is possibly due to the difference of these enzyme structures. $5 \alpha$-Redl is the rate-limiting irreversible step for the formation of many neurosteroids. Animal study suggests subsequent $3 \alpha$-reduction of dihydroprogesterone and dihydrotestosterone by $3 \alpha$-HSD into steroid metabolites which have neuroactive function via enhancing GABA suppression. These neuroactive steroids promote GABA effects by allosteric modulation at GABA-A receptors, thus exerting anticonvulsant, antidepressant, and anxiolytic effects [18]. In socially isolated mice, $5 \alpha$-Red1 is downregulated in glutamatergic pyramidal neurons that converge on the amygdala from cortical and hippocampal regions possibly causing anxiety, aggression, and cognitive dysfunction $[19,20]$.

VCZ was the weakest inhibitor for $5 \alpha$-Red1 and $3 \alpha-H S D$, with $\mathrm{IC}_{50}$ about $100 \mu \mathrm{M}$. However, VCZ almost did not inhibit $\mathrm{RDH} 2$ when $100 \mu \mathrm{M}$ was used. The reason why the potency 


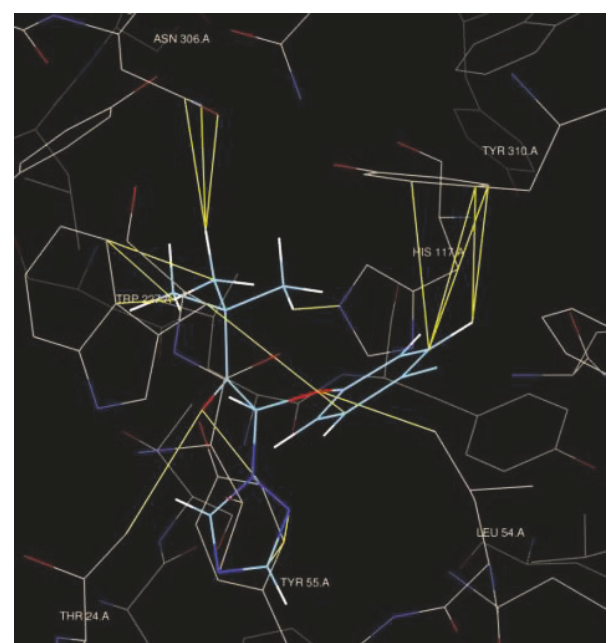

FIGURE 6: Docking analysis for the binding of triadimefon to rat's AKR1C14 (1AFS). The residues of AKR1C14 interacting with triadimefon were listed.

of VCZ is different from those of TEB and TRI is unclear. This is possibly due to the different chemical structures, in which TEB and TRI contain one triazole and VCZ contains one imidazole in the chemical structure.

TEB and TRI competitively inhibited $5 \alpha$-Red 1 when testosterone was provided. TEB and TRI also competitively inhibited $3 \alpha-$ HSD. Docking study further confirmed that these three chemicals bound to the steroid-binding pocket of $3 \alpha$-HSD. TRI interacts with Try310, Trp227, His117, Tyr55, Leu54, Thr24, and Asn306 residues in the steroid-binding pocket of $3 \alpha-$ HSD. The Tyr310 and Trp227 residues were believed to maintain stability of the steroid, and Hisl17 and Tyr55 residues of $3 \alpha$-HSD were believed to catalyze the $3 \alpha$-position of the steroid [15]. The free energy calculation further showed the lowest binding energy for TRI, which was comparable to DIOL, indicating that TRI has high affinity for $3 \alpha$-HSD.

The homeostasis of neurosteroids including ALLO and DIOL depends on the catalysis of their biosynthetic enzymes, $5 \alpha$-Red 1 and $3 \alpha$-HSD, as well as the metabolizing enzyme $\mathrm{RDH} 2$. Since $5 \alpha$-Red1 is the rate-limiting step for neurosteroid formation, this inhibition by TEB and TRI is critical for the production of neurosteroids. Indeed, evidence shows that these fungicides can affect brain function. Rats after exposure to triadimefon developed a deficit in spatial learning and reference memory [21]. Rats after perinatal exposure to tebuconazole produced neurobehavioral deficits and neuropathology [22]. Triadimefon also disrupted the transporter of extracellular dopamine, dihydroxyphenylacetic acid, homovanillic acid, and 5-hydroxyindoleacetic acid in adult rat's striatum [23]. Goldfish after acute and chronic exposure to VCZ developed dysfunction of neuroendocrine regulation of reproduction [24]. Therefore, the disruption of neurosteroid biosynthesis by these fungicides could lead to neurological dysfunction.

In conclusion, TEB and TRI are inhibitors of $5 \alpha$-Red 1 and $3 \alpha$-HSD. TEB inhibited $5 \alpha$-Redl activity more potently than the activities of $3 \alpha$-HSD and RDH2. Their negative effects on the neurosteroid accumulation were worthy of further research.

\author{
Abbreviations \\ $3 \alpha$-HSD: $3 \alpha$-Hydroxysteroid dehydrogenase \\ ALLO: Allopregnanolone \\ DHT: Dihydrotestosterone \\ DIOL: Androstanediol \\ $\mathrm{IC}_{50}$ : Half maximum inhibitory concentrations \\ RDH2: Retinol dehydrogenase 2 \\ $5 \alpha$-Red1: $5 \alpha$-Reductase 1 \\ TEB: Tebuconazole \\ T: $\quad$ Testosterone \\ TRI: $\quad$ Triadimefon \\ VCZ: Vinclozolin.
}

\section{Disclosure}

Ping Huang is a co-corresponding author.

\section{Conflicts of Interest}

The authors report no conflicts of interest.

\section{Authors' Contributions}

Xiuwei Shen and Fan Chen equally contributed to this work.

\section{Acknowledgments}

The authors thank T. M. Penning (University of Pennsylvania, Philadelphia, Pennsylvania 19104, USA) for $3 \alpha$-HSD vector. This research was supported by Health \& Family Planning Commission of Zhejiang Province (11-CX29, 2014C37017, and 2017KY466).

\section{References}

[1] X. You, Y. Li, X. Wang et al., "Residue analysis and risk assessment of tebuconazole in jujube (Ziziphus jujuba Mill)," Biomedical Chromatography, vol. 31, no. 7, p. e3917, 2017.

[2] Y. Liu, D. Shen, S. Li et al., "Residue levels and risk assessment of pesticides in nuts of China," Chemosphere, vol. 144, pp. 645-651, 2016.

[3] M. M. Frias, M. J. Torres, A. G. Frenich, J. L. M. Vidal, F. Olea-Serrano, and N. Olea, "Determination of organochlorine compounds in human biological samples by GC-MS/MS," Biomedical Chromatography, vol. 18, no. 2, pp. 102-111, 2004.

[4] L. Li, Y. Wang, X. Li et al., "Regulation of development of rat stem and progenitor leydig cells by activin," Andrology, vol. 5, no. 1, pp. 125-132, 2017.

[5] K. J. Turner, M. Morley, N. Atanassova, I. D. Swanston, and R. M. Sharpe, "Effect of chronic administration of an aromatase inhibitor to adult male rats on pituitary and testicular function and fertility," Journal of Endocrinology, vol. 164, no. 2, pp. 225238,2000 . 
[6] J. A. Zarn, B. J. Brüschweiler, and J. R. Schlatter, "Azole fungicides affect mammalian steroidogenesis by inhibiting sterol $14 \alpha$ demethylase and aromatase," Environmental Health Perspectives, vol. 111, no. 3, pp. 255-261, 2003.

[7] D. Belelli and J. J. Lambert, "Neurosteroids: endogenous regula-

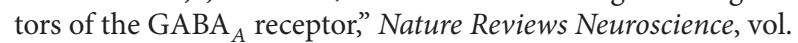
6, no. 7, pp. 565-575, 2005.

[8] D. S. Reddy, "Mass spectrometric assay and physiologicalpharmacological activity of androgenic neurosteroids," Neurochemistry International, vol. 52, no. 4-5, pp. 541-553, 2008.

[9] D. M. Berman and D. W. Russell, "Cell-type-specific expression of rat steroid 5 alpha-reductase isozymes," Proceedings of the National Academy of Sciences, vol. 90, no. 20, pp. 9359-9363, 1993.

[10] H. J. Karavolas and D. R. Hodges, "Neuroendocrine Metabolism of Progesterone and Related Progestins," in Ciba Foundation Symposium 153 - Steroids and Neuronal Activity, Novartis Foundation Symposia, pp. 22-55, John Wiley \& Sons, Ltd., Chichester, UK, 2007.

[11] N. R. Krieger and R. G. Scott, “ $3 \alpha$-Hydroxysteroid Oxidoreductase in Rat Brain," Journal of Neurochemistry, vol. 42, no. 3, pp. 887-890, 1984.

[12] X. Chai, Y. Zhai, and J. L. Napoli, "Cloning of a rat cDNA encoding retinol dehydrogenase isozyme type III," Gene, vol. 169, no. 2, pp. 219-222, 1996.

[13] D. O. Hardy, R.-S. Ge, J. F. Catterall, Y.-T. Hou, T. M. Penning, and M. P. Hardy, "Identification of the oxidative $3 \alpha-$ hydroxysteroid dehydrogenase activity of rat Leydig cells as type II retinol dehydrogenase," Endocrinology, vol. 141, no. 5, pp. 1608-1617, 2000.

[14] R.-S. Ge, H.-B. Gao, V. L. Nacharaju, G. L. Gunsalus, and M. P. Hardy, "Identification of a kinetically distinct activity of 1lbeta-hydroxysteroid dehydrogenase in rat leydig cells," Endocrinology, vol. 138, no. 6, pp. 2435-2442, 1997.

[15] M. J. Bennett, R. H. Albert, J. M. Jez, H. Ma, T. M. Penning, and M. Lewis, "Steroid recognition and regulation of hormone action: Crystal structure of testosterone and NADP+ bound to $3 \alpha$-hydroxysteroid/dihydrodiol dehydrogenase," Structure, vol. 5, no. 6, pp. 799-812, 1997.

[16] A. Grosdidier, V. Zoete, and O. Michielin, "SwissDock, a protein-small molecule docking web service based on EADock DSS," Nucleic Acids Research, vol. 39, no. 2, pp. W270-W277, 2011.

[17] E. Dong, K. Matsumoto, V. Uzunova et al., "Brain $5 \alpha$-dihydroprogesterone and allopregnanolone synthesis in a mouse model of protracted social isolation," Proceedings of the National Academy of Sciences of the United States of America, vol. 98, no. 5, pp. 2849-2854, 2001.

[18] D. A. Finn, A. S. Beadles-Bohling, E. H. Beckley et al., "A new look at the $5 \alpha$-reductase inhibitor finasteride," CNS Drug Reviews, vol. 12, no. 1, pp. 53-76, 2006.

[19] R. C. Agis-Balboa, G. Pinna, A. Zhubi et al., "Characterization of brain neurons that express enzymes mediating neurosteroid biosynthesis," Proceedings of the National Academy of Sciences of the United States of America, vol. 103, no. 39, pp. 14602-14607, 2006.

[20] R. C. Agis-Balboa, G. Pinna, F. Pibiri, B. Kadriu, E. Costa, and A. Guidotti, "Down-regulation of neurosteroid biosynthesis in corticolimbic circuits mediates social isolation-induced behavior in mice," Proceedings of the National Academy of Sciences of the United States of America, vol. 104, no. 47, pp. 18736-18741, 2007.
[21] J. Xi, Z. Yang, C. Zeng, X. Hu, and J. Wang, "Suppressive effect of triadimefon, a triazole fungicide, on spatial learning and reference memory in rats," Behavioural Pharmacology, vol. 23, no. 8, pp. 727-734, 2012.

[22] V. C. Moser, S. Barone Jr., R. J. Smialowicz et al., "The effects of perinatal tebuconazole exposure on adult neurological, immunological, and reproductive function in rats," Toxicological Sciences, vol. 62, no. 2, pp. 339-352, 2001.

[23] F. Gagnaire and J.-C. Micillino, "Effects of triadimefon on extracellular dopamine, DOPAC, HVA and 5-HIAA in adult rat striatum," Toxicology, vol. 217, no. 2-3, pp. 91-104, 2006.

[24] M. Golshan, A. Hatef, A. Zare et al., "Alternations in neuroendocrine and endocrine regulation of reproduction in male goldfish (Carassius auratus) following an acute and chronic exposure to vinclozolin, in vivo," Aquatic Toxicology, vol. 155, pp. 73-83, 2014. 

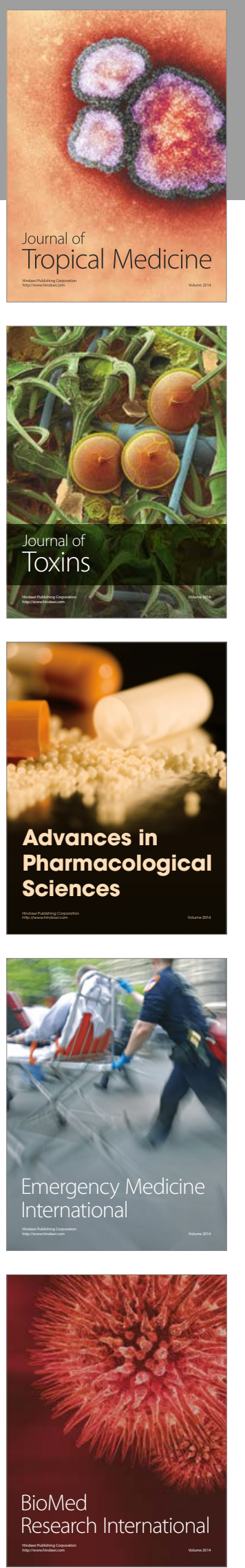
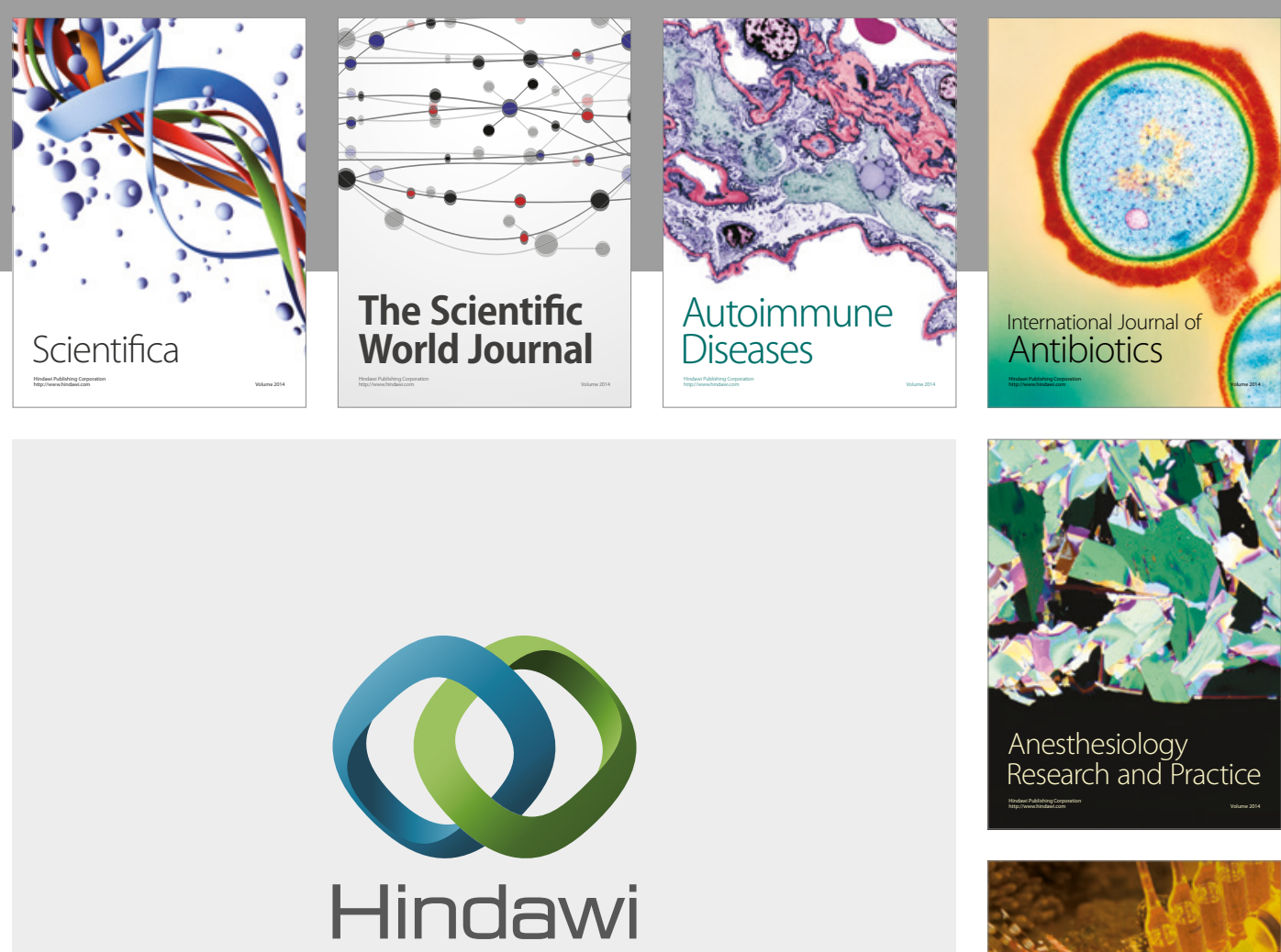

Submit your manuscripts at

https://www.hindawi.com
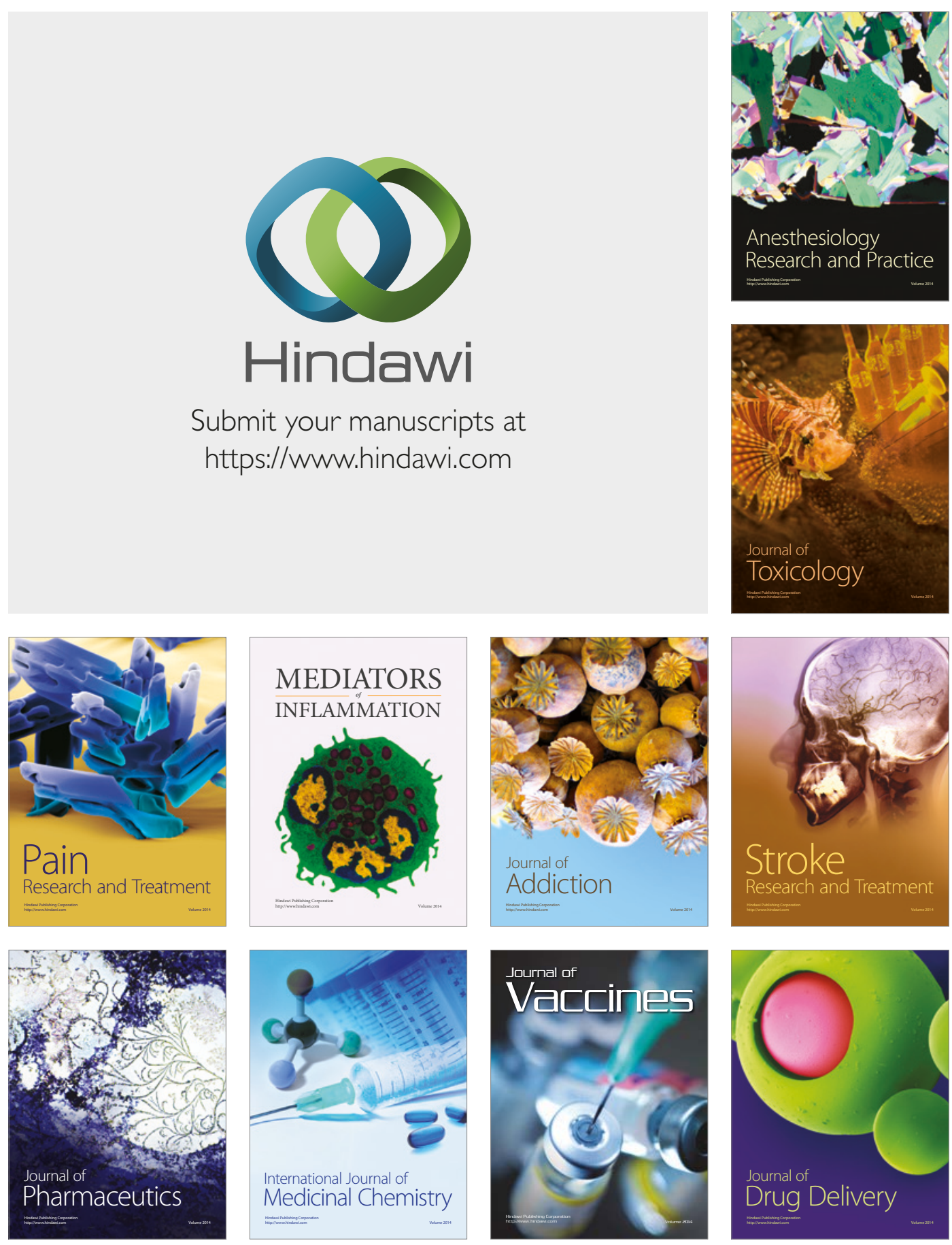